Revue des patrimoines

\title{
À la frontière de l'Égypte : les représentations du canal de Suez
}

At the frontier of Egypt, representations of the Suez canal

\section{Hélène Braeuner}

\section{(2) OpenEdition}

Journals

Édition électronique

URL : http://journals.openedition.org/insitu/20049

DOI : 10.4000/insitu.20049

ISSN : $1630-7305$

Éditeur

Ministère de la culture

Référence électronique

Hélène Braeuner, «À la frontière de l'Égypte : les représentations du canal de Suez », In Situ [En ligne], 38 | 2019, mis en ligne le 15 février 2019, consulté le 19 avril 2019. URL : http://

journals.openedition.org/insitu/20049; DOI : 10.4000/insitu.20049

Ce document a été généré automatiquement le 19 avril 2019

\section{cc) $(7)$}

In Situ Revues des patrimoines est mis à disposition selon les termes de la licence Creative Commons Attribution - Pas d'Utilisation Commerciale - Pas de Modification 4.0 International. 


\title{
À la frontière de l'Égypte : les représentations du canal de Suez
}

\author{
At the frontier of Egypt, representations of the Suez canal
}

\author{
Hélène Braeuner
}

1 Le canal de Suez, ouvert entre la mer Méditerranée et la mer Rouge au terme de dix années de travaux de creusement (1859-1869), transforme en profondeur ce territoire aux confins de l'Égypte. Il n'est pas à proprement parler la limite géographique au nord-est du pays, la péninsule du Sinaï bordant la frontière avec la Palestine. Mais l'isthme de Suez, une fois percé, fait apparaitre une nouvelle ligne de démarcation, une séparation qui porte toutes les caractéristiques de l'espace frontalier. Objet de rapports de force entre différentes entités, favorable aux échanges comme à l'affirmation de pouvoirs, il cristallise les plus intenses rivalités et représente une limite géographique entre deux mondes qui a fait naître de nombreuses convoitises. Paradoxalement, cette « limite », loin d'être infranchissable, facilite la circulation des navires entre l'Occident et les Indes. Le canal devient une porte de l'Orient, un passage, une voie de communication. Pour les Égyptiens, il désigne une région en marge du pays, dominée par les étrangers. Pour les Occidentaux, il est un espace créé en terre orientale, un lieu désertique qui se transforme en région hospitalière grâce au génie français et aux innovations techniques commandées par la Compagnie universelle du canal maritime de Suez. Il constitue, de Port-Saïd à Suez, un territoire à part dans la géographie et l'histoire de l'Égypte où apparaissent des éléments architecturaux spécifiques dans les villes créées sur son parcours ${ }^{1}$. Un corpus d'images inédites se forme dès le chantier de creusement, témoignant du nouveau visage qui se dessine dans l'isthme, révélant de nouveaux motifs dans la production artistique orientaliste et portant les traces d'échanges propres aux territoires partagés.

\section{La limite et l'au-delà}

2 Le percement du canal de Suez est un épisode extraordinaire de l'histoire des progrès techniques de l'Europe industrielle qui imprime une nouvelle physionomie à la frontière orientale de l'Égypte. La modernité du site marque une rupture avec le voyage 
traditionnel de la première moitié $\mathrm{du} \mathrm{XIX}^{\mathrm{e}}$ siècle: «C'est sur cette terre ancienne qu'apparaît le signe d'une modernité qui contribue à mettre fin à l'orientalisme traditionnel, à un moment où l'occidentalisation est galopante ${ }^{2}$. " Les peintres dessinent l'image d'une nouvelle Méditerranée où la modernité impose ses chantiers ${ }^{3}$. Mais le canal de Suez, est-ce encore l'Égypte? Il coupe l'isthme qui représentait lui-même la limite du continent africain, la péninsule égyptienne du Sinaï se situant géographiquement en Asie.

Longtemps, le territoire de l'isthme de Suez n'est pas intégré au circuit de visite égyptien. Il faut du temps pour que les voyageurs conçoivent une autre Égypte que celle des temples pharaoniques. On a souvent voulu l'isoler, comme dans la Description de l'Égypte où il est le territoire par lequel le pays se termine. Pour certains voyageurs, se rendre sur le canal de Suez, c'est déjà quitter l'Égypte, qui se borne aux deux rives du Nil. Le zoologue Geoffroy Saint-Hilaire, qui participe à la campagne de 1798, n'envisage pas le territoire autrement: "Salahieh est le point le plus éloigné du Caire sur la route de Damas, c'est là que de ce côté, l'Égypte est terminée ; au-delà commence l'isthme de Suez ${ }^{4}$ .» Cette «fin» de l'Égypte a généré des représentations différentes de l'orientalisme traditionnel qui se nourrit de l'Égypte millénaire. Elle prend tour à tour l'image d'une route, d'un passage, d'une frontière géographique ou symbolique qui n'est pas sans rappeler les particularités de l'insularité dans les romans d'aventures et les récits de voyage ${ }^{5}$.

4 Le creusement du canal, en agrandissant l'Égypte, donne ensuite au voyageur un espace supplémentaire à apprivoiser. Le voyageur est d'abord fasciné par le changement de décor, la colonisation du désert, la création de villes nouvelles. On y voit une résonance de la foi saint-simonienne : la croyance dans le progrès accompagne les premières œuvres des artistes du canal. Ils célèbrent la vitalité nouvelle du pays et l'intervention de la modernité. C'est un Orient lessepsien qui s'impose et pour lequel on délaisse le pittoresque du Nil et les bazars du Caire pour faire « l'école buissonnière de Port-Saïd à Suez ${ }^{6} »$. On fait des visites émerveillées dans les ateliers de forge, les bassins et les usines. Il faut avoir vu les milliers d'Égyptiens au travail, les remorqueurs et les dragues charriant le sable sur les berges. Il faut être allé jusqu'aux fontaines de Moïse en ayant visité le chalet Lesseps à Ismaïlia et la maison de Bonaparte à Suez. Ces nouveaux motifs acquièrent, dans les souvenirs et les carnets de croquis, l'importance de «monuments ». Les jardins font aussi partie des incontournables, l'explorateur irlandais Thomas Kerr Lynch (1818-1891) cite déjà en 1866 ceux d'Ismaïlia et d'El-Guisr7.

5 Le canal de Suez a ensuite constitué une zone bien spécifique du voyage oriental, imprégné de l'idée de passage entre deux mers et d'ouverture vers un espace plus lointain. Il a été un temps la limite du voyage en Orient avant que l'Égypte ne se réduise à une étape précédant des destinations plus lointaines. On associe à la réalité du canal le mythe de l'ouverture à un autre monde, celui du rapprochement des civilisations et celui du progrès. De l'isthme au canal a émergé comme une symbolique des possibles: tout l'avant-chantier est porteur de prophéties, d'espérance, annonciateur d'un espace sans limites, offrant le vertige de l'au-delà. Certaines images traduisent l'intégration de l'isthme à l'Égypte traditionnelle, d'autres sortent le territoire de l'ensemble commun des représentations du pays. Inexorablement, les récits annoncent que l'Égypte devient trop proche du monde occidental et la réduisent à un passage, qui entraine du côté des Indes, promesse de plus de dépaysement. 


\section{La tranchée et la ligne d'eau, nouveaux motifs de l'isthme}

6 Le canal de Suez possède les caractéristiques symboliques de figuration de la frontière. De nombreuses images (gravures dans la presse illustrée, dessins et peintures) illustrent ces motifs spécifiques évoquant la limite, le passage, l'autre côté. Les vues à vol d'oiseau du tracé du canal de Suez dévoilent la ligne d'eau qui sépare le territoire en deux ${ }^{8}$. Narcisse Berchère (1819-1891), l'un des artistes missionnés par la Compagnie pour peindre en 1862 la première phase de construction du canal, fait apparaître dans son album un panorama de l'isthme de Suez annoté du nom des stations, des routes, des campements nouveaux créés tout le long du parcours (fig. 1).

Figure 1

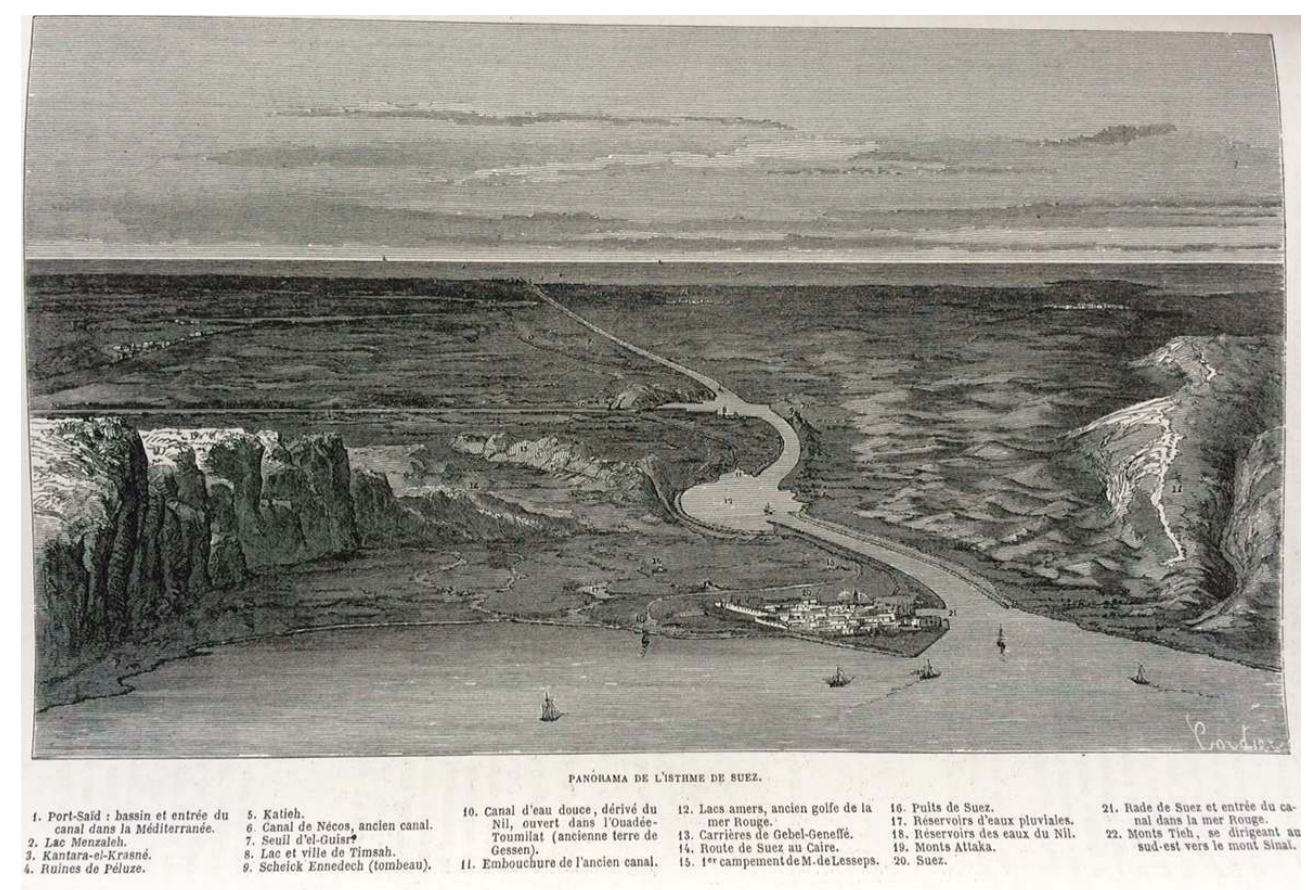

«Panorama de l'isthme de Suez ». Dessin de Dominique Grenet tiré de l'album inédit de Narcisse Berchère et publié dans MERRUAU, Paul. « Une excursion au canal de Suez ». Le Tour du Monde, Nouveau journal des voyages, 1862, p. 4.

Droits réservés.

7 Une nouvelle géographie naît avec le canal pour lequel sont fondées deux villes nouvelles, Port-Saïd, à l'extrémité occidentale, et Ismaïlia, en son milieu, sur les Lacs amers. François Barry, envoyé la même année par la Compagnie, sent combien il importe de montrer le bouleversement physique qui s'opère sur ces terres désertiques. Son huile sur toile intitulée LeChantier $n^{\circ} 5$ : vue $d u$ canal de Suez montre les proportions impressionnantes de la tranchée en cours d'exécution dans le seuil d'El-Guisr (fig. 2). Les campements apparaissent au loin, de l'autre côté de la voie d'eau. Les rives dénudées sont le sujet du tableau, se dévoilant d'un côté dans l'ombre du massif rocheux que l'on a creusé et, de l'autre, révélant une blancheur de craie dans la lumière du soleil levant. Le peintre dresse le portrait d'un espace entre deux mondes où cohabitent le motif 
traditionnel d'une dahabieh ${ }^{9}$ halée par un chameau au premier plan et la modernité d'un élévateur de déblais sur les hauteurs de la berge qui annonce la mécanisation prochaine du chantier.

Figure 2

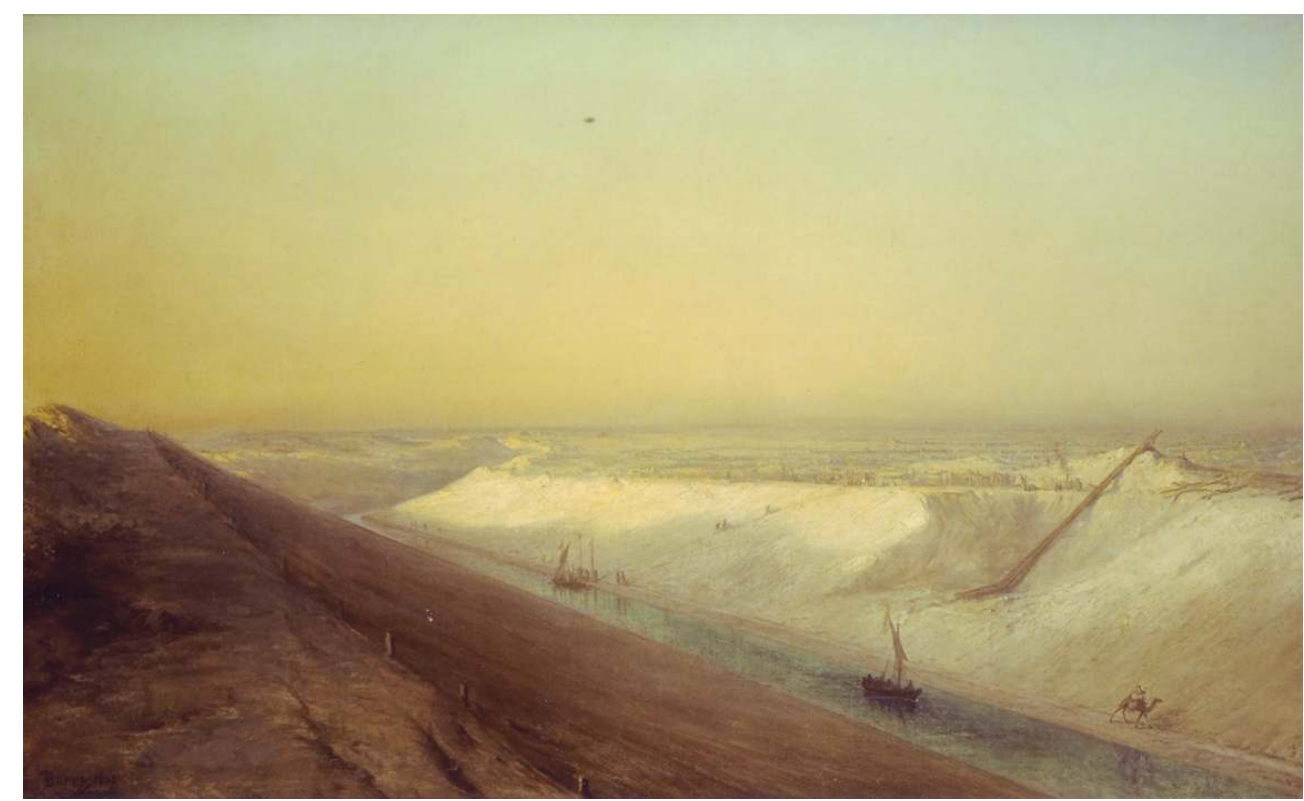

BARRY, François. Le Chantier no 5 : vue du canal de Suez. 1863, huile sur toile, Paris, Souvenir de Ferdinand de Lesseps et du canal de Suez.

Droits réservés.

$8 \quad$ La symbolique de la coupure apparaît de plus en plus dans les récits de voyageurs visitant les travaux de percement. Le vocable n'est pas nouveau dans l'histoire de la réunion des deux mers qui remonte au canal des Pharaons. Un bas-relief gravé sur le mur septentrional extérieur du temple d'Amon de Karnak montre le canal de Sésostris $\mathrm{I}^{\mathrm{er}}$ (1971-1926 av. J.-C.) reliant à cette époque le bras le plus oriental du Nil à la mer Rouge. Les égyptologues Georges Ebers et Gaston Maspero $^{10}$ rapportèrent «l'apparente simplicité » de la composition, "d'une signification et d'une importance exceptionnelles »: « Nous y voyons le conquérant; il revient vainqueur de Syrie, et les prêtres et les nobles d'Égypte l'accueillent à grand renfort de bouquets et de compliments, sur la rive du Canal. Le Canal est défendu par des fortifications et porte dans une inscription le nom de Coupure ${ }^{11}$. Quand l'avancée des travaux pharaoniques menés par Ferdinand de Lesseps annonce un proche succès, la coupure devient une réalité physique. Il faut décrire autrement cette géographie nouvelle et la tranchée est présentée, par les partisans du canal, comme une réparation de la nature. Théophile Gautier, qui résiste pourtant au discours euphorique sur la célébration du mariage de l'Orient et de l'Occident, utilise un vocabulaire thérapeutique et parle du canal de Suez comme d'une agrafe entre les deux mers ${ }^{12}$ - celui de Panama sera plus tard un " point de suture ». L'écrivain découvre alors l'impressionnant plan-relief du Pavillon de l'isthme construit par la Compagnie à côté du parc égyptien de l'égyptologue Auguste Mariette à l'Exposition universelle de 1867, à Paris. C'est devant cette immense maquette que Ferdinand de Lesseps organise ses nombreuses conférences pour rendre compte des remarquables progrès du chantier ${ }^{13}$. C'est d'ailleurs la figure du grand Français, comme le nomme plus tard Gambetta, qui apparait dans les représentations symbolisant la 
séparation des terres. Pour ouvrir «son» canal, il pousse littéralement les continents. Dans la caricature d'Étienne Carjat (fig. 3), moqué en Hercule athlétique, il enjambe le canal où passent des navires, en écartant puissamment les roches de ses bras. De part et d'autre de cette nouvelle frontière créée par la force des hommes se trouvent figurés le crocodile, symbole de l'Égypte antique associé au Nil, et une autre figure de la mythologie égyptienne, le sphinx couchant. L'un et l'autre motifs, soudain séparés par la percée de l'isthme, font sentir combien la division est subie au sein d'un même territoire. Pour l'italien Casimiro Teja (1830-1897), qui rapporte un album illustré de caricatures retraçant les épisodes observés pendant le voyage d'inauguration du canal en novembre $1869^{14}$, Ferdinand de Lesseps a littéralement scié les deux continents et séparé l'Afrique et l'Asie. Le canal de Suez qui fut largement promu et légitimé dans ses discours, ceux des membres de la Compagnie et ceux des partisans du projet comme devant réunir deux mers et annuler la frontière entre deux mondes, finit par en incarner une lui-même.

Figure 3

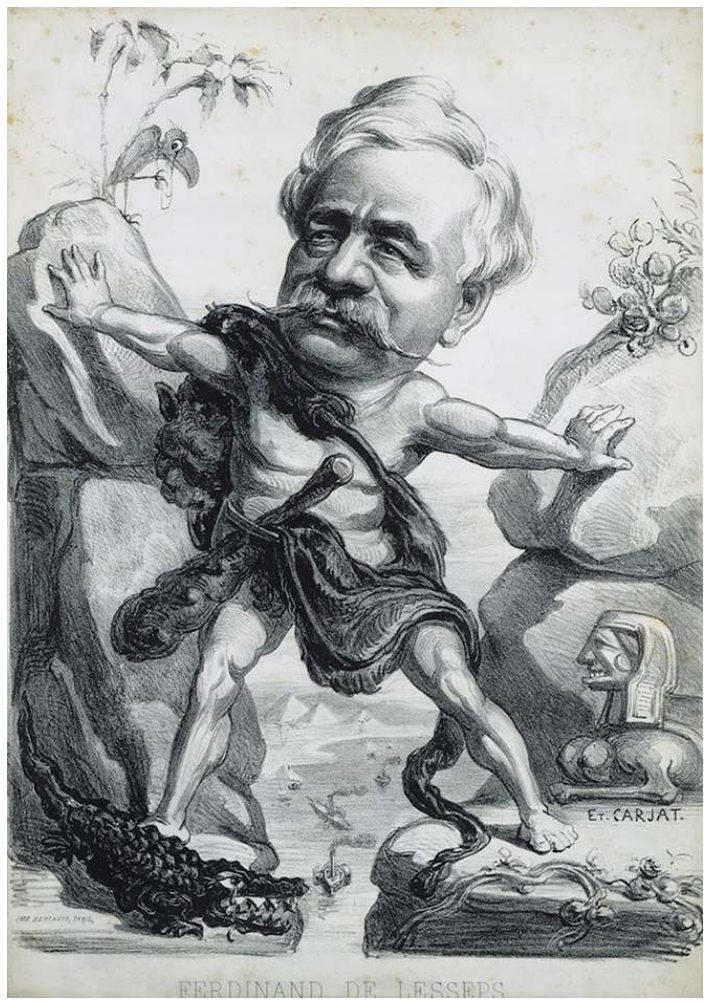

CARJAT, Étienne. Portrait de Ferdinand de Lesseps. Gravure, Paris, Souvenir de Ferdinand de Lesseps et du canal de Suez.

Droits réservés.

9 La quête de la réunion des peuples s'est doublée physiquement de la conquête d'un espace. Edward Said (1935-2003) a plus tard dénoncé la fonction d'appropriation derrière le projet de percement de l'isthme et souligné avant tout un changement de nature tout à fait inédit. Il évoque cette barrière de terre transmuée en une artère liquide et la compare au changement de substance de l'Orient, passant «d'une résistance hostile à une association obligeante et soumise $»^{15}$. La frontière terrestre entre les deux mers se transforme en une frontière maritime entre deux terres qui porte la marque de 
l'étranger. Mais le canal ne sépare pas deux pays différents: les motifs de l'Occident apparaissent sur le tracé même de cette nouvelle ligne de démarcation.

\section{Images de conquête et de triomphe}

$10 \mathrm{Au}$ cours des travaux de percement de l'isthme, des lignes de chemin de fer sont construites pour acheminer le matériel de construction, les campements de Port-Saïd et Ismaïlia sont remplacés par des immeubles aux architectures occidentales, le chantier est réalisé grâce à des contingents égyptiens avant que ceux-ci soient remplacés par des machines de creusement fabriquées à Marseille. Durant les dix années que dure le chantier, ce qui est en jeu dans cette figuration du canal de Suez, c'est l'affirmation d'une conquête géographique et d'une victoire scientifique et technique. Les images montrent un triomphe, celui des machines qui dominent et disciplinent la terre, celui d'une entreprise humaine et éclairée qui a été capable de transformer un espace désertique en une région hospitalière.

Les gravures publiées dans la presse illustrée à partir du chantier de creusement marquent le début d'une iconographie qui va exploiter l'image d'une entreprise de pionniers, à l'instar des migrants de l'Ouest américain ${ }^{16}$. Le sculpteur Auguste Bartholdi (1834-1904), tandis qu'il séjourne en Égypte pour soumettre son projet de statue-phare au vice-roi ${ }^{17}$, décrit Port-Saïd comme une ville "avec toutes ses maisons, ses huttes ou baraques alignées sur de larges rues, dans le sable, [qui] représente bien les villes comme on se les figure créées par les pionniers américains ${ }^{18}$. Les hommes de la Compagnie universelle du canal maritime de Suez et les ouvriers du chantier sont montrés colonisant un territoire vierge et inexploré, repoussant les limites physiques de la géographie du lieu 19. CEuvrant au marquage d'un nouveau sillon, ils sont les «pionniers de l'avenir » dans la préface en vers du recueil du comte Louis de Trogoff de Kerbiguet, «bataillon de travailleurs » mené par un Ferdinand de Lesseps chef de guerre dans l'isthme « rebelle $»^{20}$ . Le bouleversement du territoire s'annonce aussi dans les images de dynamitage des obstacles rocheux ${ }^{21}$. Le panorama d'El-Guisr dévoile l'établissement des premières maisons, les cabanes de chantier et l'église, qui viennent peupler un horizon encore désertique à peine animé par la circulation de quelques groupes de chameaux à l'allure bien pittoresque. La construction du chantier à Port-Saïd est spectaculaire tandis que s'installe tout un attirail de caisses, d'outils, de pièces de machines, de stocks de marchandises, de rails, d'aménagement d'appontements et de quais pour le déchargement de tous les nouveaux équipements qui débarquent sur le sol de l'isthme encore vierge. Ces nouveaux motifs sont comme des avant-postes placés sur une ligne frontière.

12 La rupture est encore plus marquante avec l'apparition des machines de creusement, largement décrites dans les reportages publiés dans la presse illustrée ${ }^{22}$ et qui ne manquent pas de fasciner les visiteurs du canal. Avec Émile de La Bédollière (1812-1883), journaliste délégué aux fêtes d'inauguration du canal,

nous admirons là les dragues colossales de MM. Borel et Lavalley, qui, prenant la terre dans le lit du canal, la montent à la cime des berges et la déversent sur le désert. Elles ont remué chaque mois, depuis plusieurs années, une moyenne de quatorze millions de mètres cubes de terres. Leur service a exigé, par mois, vingtdeux mille employés, dix mille tonnes de charbon, vingt-six mille kilogrammes d'huile. Pour avoir une idée de la masse de déblais qu'ils ont mise en mouvement, que l'on s'imagine, a écrit l'ingénieur Cadiat, l'avenue des Champs-Élysées couverte 
dans toute sa longueur d'une montagne de cent mètres de base et de vingt-huit mètres de hauteur au sommet. Qu'on imagine encore la place Vendôme couverte de terre jusqu'à quatre fois la hauteur des maisons qui la bordent ${ }^{23}$. mère le portrait à l'aquarelle d'une drague à long couloir (fig. 4) et Edmond About (1828-1885), dans Le Fellah ${ }^{24}$, reconnaît que ces « grands engins de l'industrie ne sont pas pittoresques comme le Parthénon » mais les proportions gigantesques de certaines de ces structures de fonte et de fer lui font penser à des cathédrales.

Figure 4

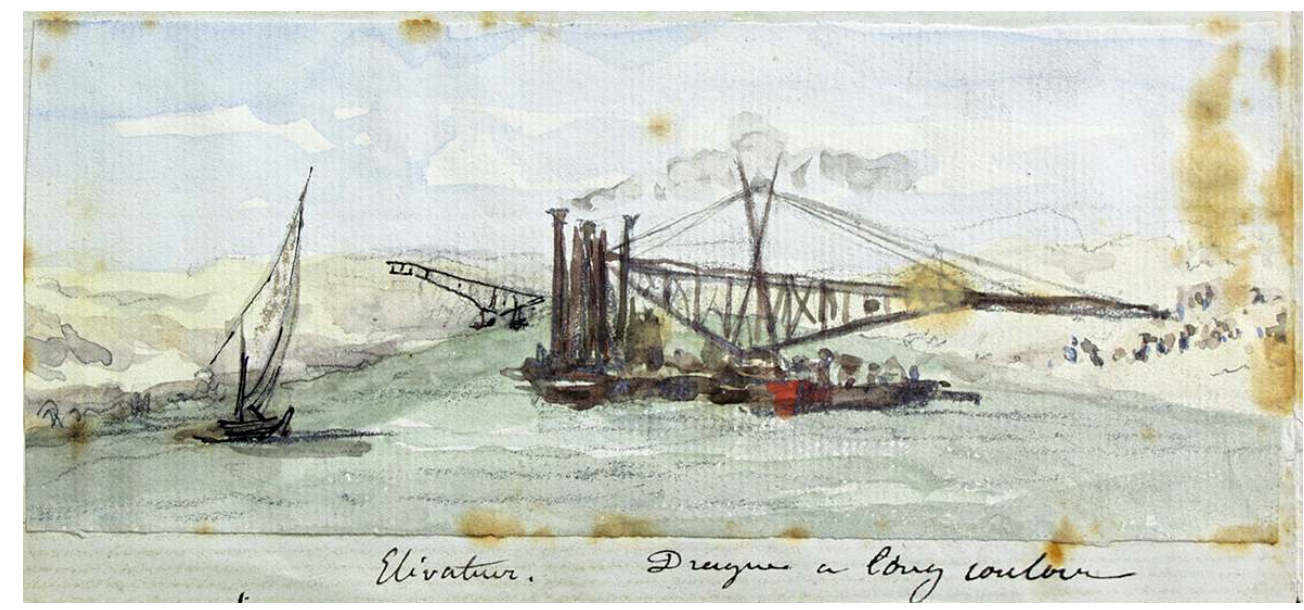

BARTHOLDI, Auguste. «Élévateur. Drague à long couloir ». Aquarelle. Dans Lettres et journal du second voyage en Égypte (1869) d'Auguste Bartholdi adressés à sa mère, 13 avril 1869, Colmar, musée Bartholdi, cote VI $3 \mathrm{E}$.

Droits réservés.

Sur cette terre qui se colonise, les artistes se laissent séduire par ces motifs industriels et ces architectures importées. Le progrès technique domine le chantier et modèle la terre avec plus de brutalité qu'à l'époque des fellahs. On finit par oublier la physionomie de l'isthme sans guère éprouver de nostalgie : «Qu'était le désert entre Port-Saïd et Suez avant que M. de Lesseps y eût jeté les germes de la civilisation en y faisant serpenter le bleu ruban que forme le bienfaisant canal $?^{25}$ » Par contre, le voyageur regrette de plus en plus de n'y croiser que ses compatriotes et finit par rechercher les détails pittoresques de l'Orient dans les quartiers du vieux Suez. Aux marges de l'Égypte, le canal de Suez porte le caractère de l'autre et de l'étranger.

\section{La mémoire en partage}

Le chantier de percement de l'isthme est dirigé par une entreprise française en terre égyptienne, avec une main d'œuvre d'abord indigène puis internationale. La population des villes du canal est proche de l'image cosmopolite de Constantinople, avec une carte des nationalités qui lui est propre. L'immense tranchée rêvée par Ferdinand de Lesseps, axe de circulation, voie de passage, est devenue un territoire partagé qui a produit un patrimoine singulier. Le journaliste Émile de La Bédollière est marqué par le caractère international des villes du canal. La rue François-Joseph, artère marchande d'Ismaïlia, présente des enseignes dans toutes les langues : Tobaco e sigari, Notice to travellers, Au vieux zouave, Rose, marchand de vins... Le canal de Suez porte les traces d'échanges et le mélange 
de cultures propres aux espaces de frontière. La rencontre des nations, largement popularisée par les discours de légitimation du projet, est-elle une réalité transmise par les imagiers du canal de Suez?

Figure 5

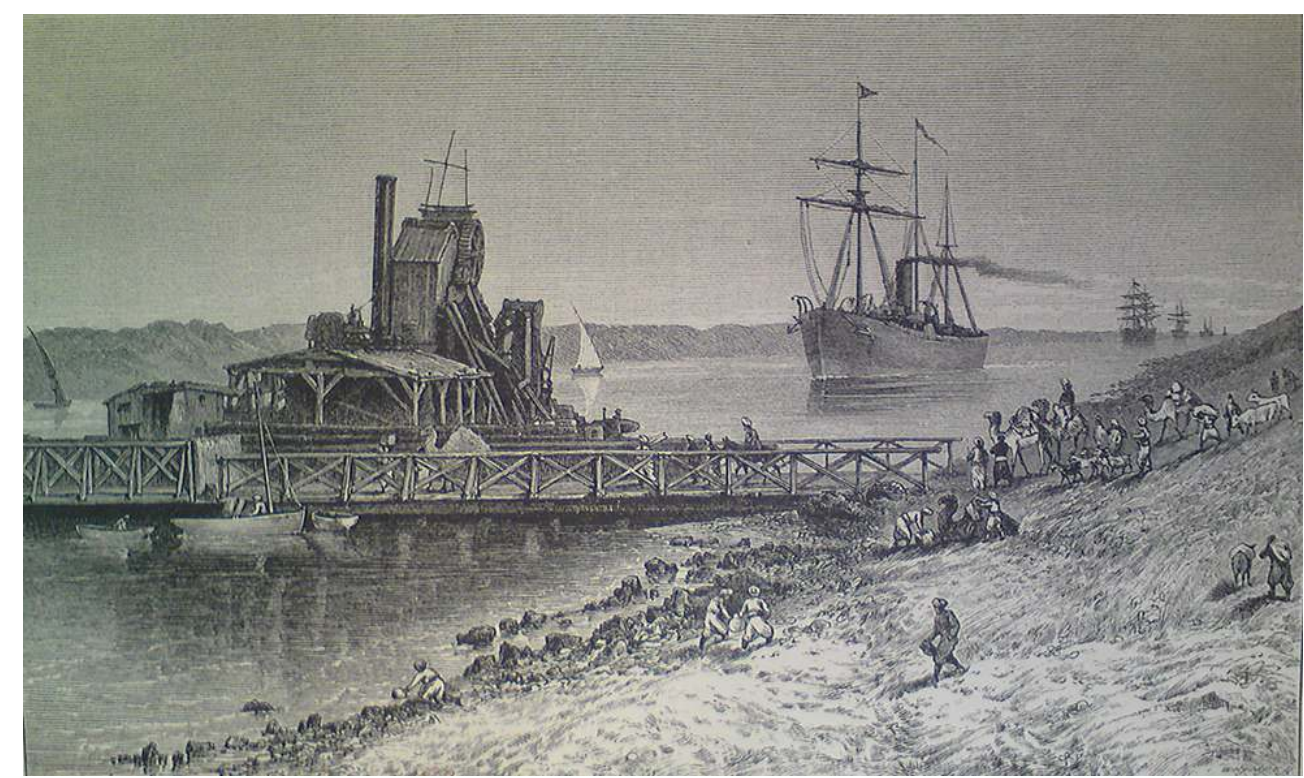

«View of Kantara ». Dans VILLIERS STUART, Henry Windsor. Egypt after the War. Being the Narrative of a tour of inspection (undertaken last autumn)... With Numerous coloured plates and woodcuts. Londres : 1883, p. 491.

Droits réservés.

La Vue de Kantara (fig. 5) publiée par le britannique Henry Villiers Stuart (1827-1895) semble s'interroger sur les contrastes que porte cet espace: au premier plan, l'embarcadère des caravanes en route pour la Syrie, l'endroit, selon l'auteur, où passèrent Joseph et la Vierge Marie ; derrière, une machine de dragage et, dans le fond, une file de steamers assurant le lien entre l'Orient et l'Occident. C'est l'image de deux mondes qui se croisent et qui ont chacun leur sens de circulation, sans qu'ils semblent se voir. C'est à cet endroit que passent toutes les caravanes qui viennent de Basse-Égypte ou de la Tripolitaine pour se rendre en Syrie ou en Arabie. C'est toujours le point d'arrêt d'une foule de Bédouins et d'Arabes qui égayent la traversée des voyageurs, avec leurs longues files de chameaux chargés de marchandises. Le photographe Hippolyte Arnoux, installé à Port-Saïd à partir du chantier de percement, s'intéresse à l'exploitation maritime du canal et diffuse la même image du canal, observé comme une frontière et un obstacle à franchir (fig. 6). "Ce tableau est plein de couleur locale; les Arabes barbus, à l'air farouche, couverts de la poussière du désert, drapés à l'antique; les chameaux à la démarche lente, tout cela, sous le ciel pur de l'Égypte, a beaucoup de caractère ${ }^{26}$ » écrit le fonctionnaire colonial Adrien Domergue, en route pour Madagascar. Les files d'Orientaux prennent des allures de caravanes bibliques plus séduisantes que le spectacle d'une locomotive dans le désert sur le chemin de fer du Caire à Suez. On imagine pourtant combien le percement du canal change jusqu'aux habitudes de circulation des Arabes vers la péninsule Arabique. Les voies terrestres de communication qui avaient une histoire millénaire ont été coupées avec le canal de Suez ${ }^{27}$. Les Bédouins, acteurs silencieux de cet 
épisode historique, disparaissent non seulement des catégories statistiques mais aussi des représentations.

Figure 6

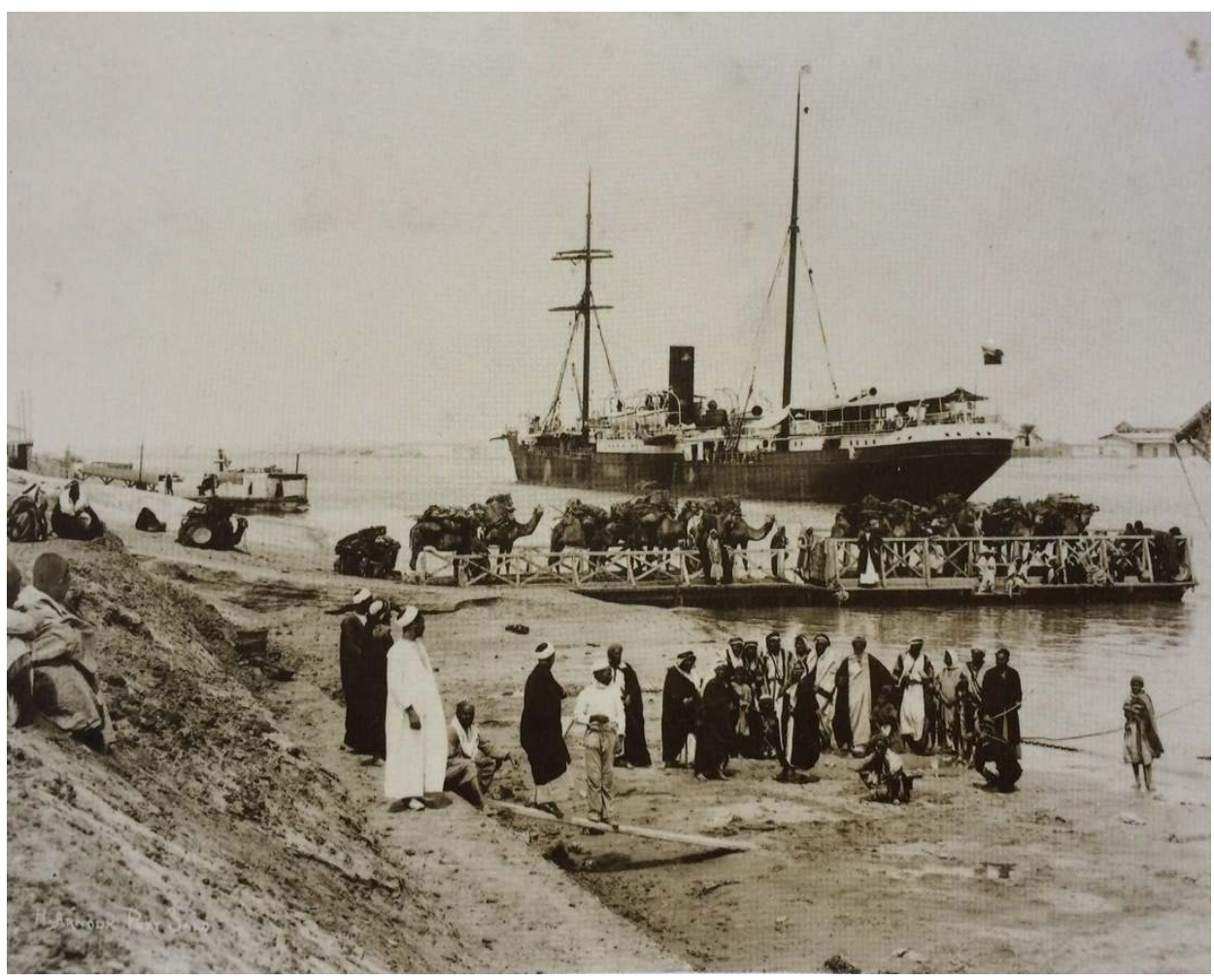

ARNOUX, Hippolyte. Kantara. Km 45. Bac des chameaux et navires en transit. Tirage photographique sur papier albuminé, entre 1870 et 1890, Roubaix, Archives nationales du monde du travail.

(c) Archives nationales du monde du travail.

17 Comment ce territoire en partage a-t-il accueilli les ambitions de commémoration des acteurs du canal de Suez? La réunion des deux mers ne voit pas émerger d'œuvres collectives. De nombreux monuments jalonnent le parcours mais ils sont soit commandés par la Compagnie du canal de Suez (la statue de Ferdinand de Lesseps par Emmanuel Frémiet à Port-Saïd (fig. 7, fig. 8), inaugurée en 1899) soit le symbole des rivalités qui persistent même dans les monuments rendant hommage aux troupes françaises et anglaises ayant défendu le canal pendant la Première Guerre mondiale ${ }^{28}$. Leur alignement tout au long du parcours semble figurer une sorte de déplacement de la frontière francobritannique en terre égyptienne. Le phénomène de patrimonialisation de la figure du créateur du canal et de son œuvre ${ }^{29}$ n'a pas manqué de cristalliser les rancœurs des Égyptiens - le monument en bronze de Frémiet fut dynamité au cours de la crise de 1956. Les enjeux de ce processus mémoriel et son instrumentalisation se sont confrontés aux ambitions de la mémoire égyptienne dont le regard sur cette histoire commune diffère fondamentalement ${ }^{30}$. 


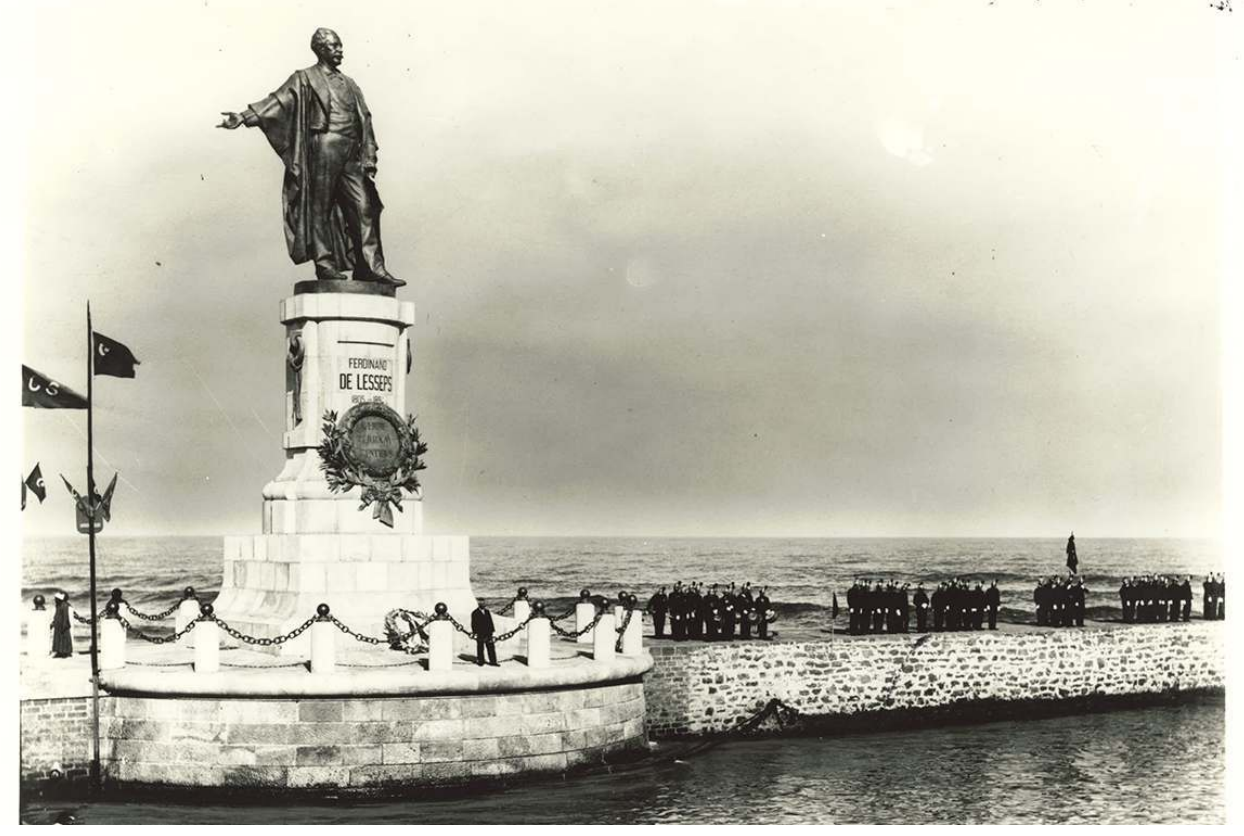

Inauguration de la statue de Ferdinand de Lesseps à l'entrée de Port-Saïd. 1899, photographie, Paris, Souvenir de Ferdinand de Lesseps et du canal de Suez.

Droits réservés.

Figure 8

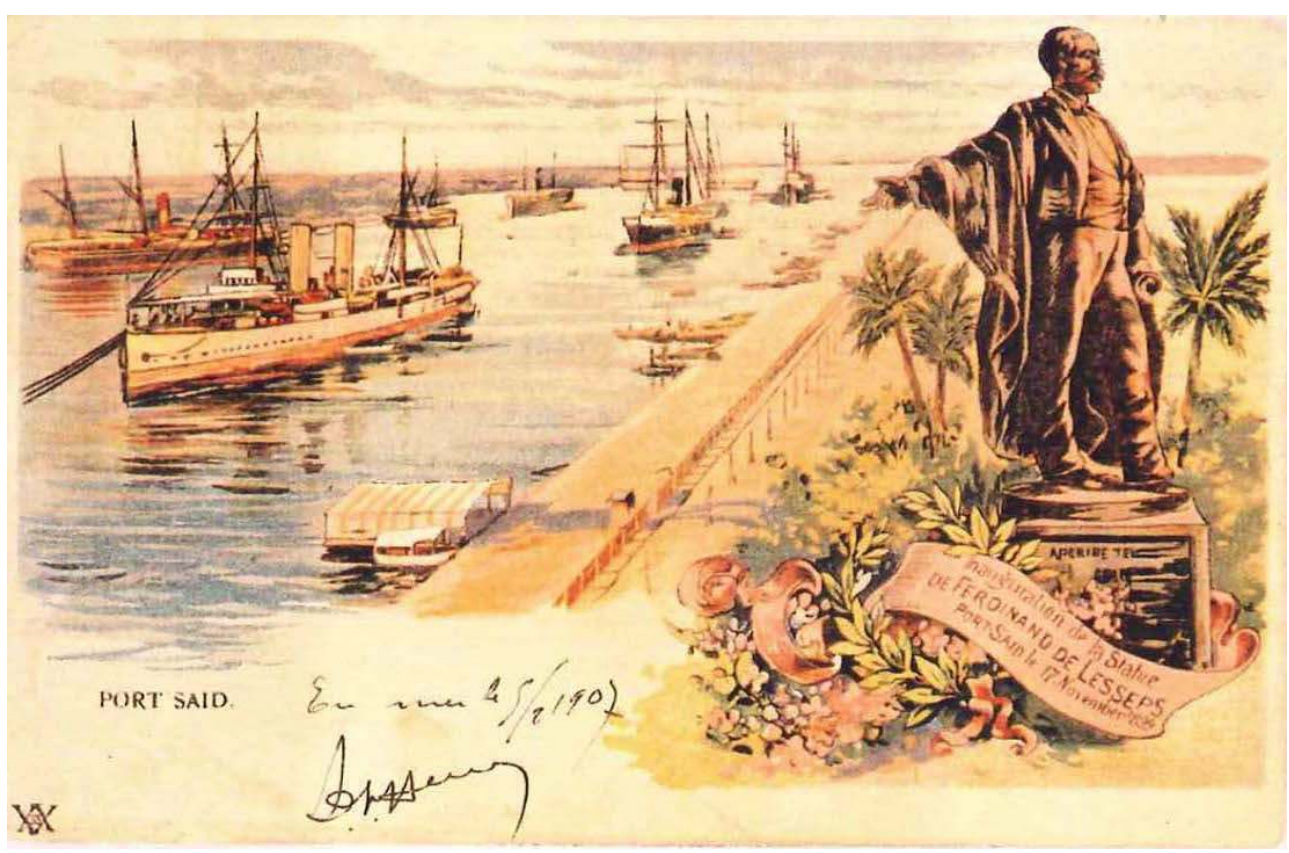

Inauguration de la statue de Ferdinand de Lesseps à l'entrée de Port-Saïd. Carte postale, Paris, Souvenir de Ferdinand de Lesseps et du canal de Suez.

Droits réservés.

Récemment, en plein contexte de doublement du canal, un projet égyptien de redressement de la statue de Lesseps avait été annoncé par le gouverneur de Port-Saï : le 
grand Français devait reprendre sa place sur son piédestal tandis que sur le quai seraient érigées une statue de Nasser et une autre d'un fellah ${ }^{31}$. Le canal de Suez est une fierté nationale, souvent célébré dans l'espace public comme sur les grandes fresques ornant les murs de l'Académie militaire du Caire et illustrant les richesses de l'Égypte. Dans le reste du monde, il continue d'être un sujet de création artistique, depuis le pont flottant de Christo, en 1967, projetant une réconciliation symbolique entre l'Égypte et Israël ${ }^{32}$ jusqu'à des expressions plus intimes de la traversée, qui ne manque jamais d'impressionner ${ }^{33}$.

\section{NOTES}

1. - CROSNIER LECONTE, Marie-Laure, GHITANI, Gamal, AMIN, Naguib. Port-Saï, architectures XIX ${ }^{e}$ $X X^{e}$ siècles. Le Caire: Ifao, 2006; PIATON, Claudine (dir.). Ismailia: architectures $\mathrm{XIX}^{e}-\mathrm{XX}^{e}$ siècles. Le Caire : Ifao, 2009 ; Ead. (dir.). Suez, histoire et architecture. Le Caire : Ifao, 2011.

2. - PELTRE, Christine. Dictionnaire culturel de l'Orientalisme [2003]. Paris : Hazan, 2008, p. 47.

3. - Ead. Orientalisme. Paris : Terrail, 2004, p. 182.

4. - GEOFFROY SAINT-HILAIRE, Étienne. «Lettre XXII. Au citoyen Geoffroy père, à Étampes, Maison de Cassin-bey, 13 vendémiaire an VII [jeudi 4 octobre 1798]». Dans Id. L'Expédition d'Égypte, 1798-1802. Clermont-Ferrand: Paleo, 2000, p. 63.

5. - RACAULT, Jean-Michel. "Avant-propos ». Dans MARIMOUTOU, Jean-Claude et RACAULT, Jean-Michel (éd.). L'Insularité, thématique et représentations. Actes du colloque international de Saint-Denis de la Réunion, avril 1992. Paris : L'Harmattan, 1995, p. 9-13.

6. - BAILLIÈRE, Henri. En Égypte. Alexandrie, Port-Said, Suez, Le Caire. Journal d'un touriste. Paris : J.-B. Baillière \& fils, 1867, p. 197.

7. - LYNCH, Thomas Kerr. A Visit to the Suez Canal. Londres : Day and Son, 1866.

8. - « Vue à vol d'oiseau de l'isthme de Suez ». Dessin de Oscar Mac Carthy. Le Magasin pittoresque, décembre 1858, t.XXVI, p. 401, voir le site: https://gallica.bnf.fr/ark:/12148/bpt6k31441h/ f405.item.r=suez [consulté le 31/01/2019]. «Vue panoramique du percement de l'isthme de Suez ». Le Monde illustré, 20 novembre 1858, p. 333, voir le site : https://gallica.bnf.fr/ark:/12148/ bpt6k64582244/f13.image.r=suez [consulté le 31/01/2019] ; RIEGER, Alberto. Il Canale di Suez, 1864, huile sur toile, Trieste, musée Revoltella.

9. - Bateau traditionnel égyptien à voile.

10. - EBERS, Georg. L'Égypte, 1. Alexandrie et Le Caire, 2. Du Caire à Philae [1879]. Traduction de Gaston Masper. Paris : Firmin-Didot, 1880-1881.

11. - Cité par CHARLES-ROUX, Jules. L'Isthme et le canal de Suez. Historique, état actuel. Paris: Hachette, 1901, p. 14.

12. - GAUTIER, Théophile. «L'isthme de Suez ». Le Moniteur universel, $\mathrm{n}^{\circ} 215,3$ août 1867.

13. - BLANCHARD, Auguste. "Conférence de Ferdinand de Lesseps devant la maquette du canal de Suez ». Gravure sur bois pour L'Illustration, journal universel, 28 septembre 1867.

14. - L'album Pasquino all'Istmo di Suez était conçu comme un cadeau destiné aux abonnés de l'hebdomadaire satirique Il Pasquino en 1870.

15. - SAID, Edward W. L'Orientalisme. L'Orient créé par l'Occident [1978]. Paris: Seuil, 1997, rééd. 2003, p. 110.

16. - Voir GAUTHIER, Guy. Édouard Riou, dessinateur. Entre le "Tour du Monde " et Jules Verne, 1860-1900. Paris: L'Harmattan, 2008, p. 15. Voir également le catalogue de l'exposition par 
SALOMÉ, Laurent (dir.). La Mythologie de l'Ouest dans l'art américain, 1830-1940. Rouen, musée des Beaux-Arts, Rennes, musée des Beaux-Arts, Marseille, Centre de la Vieille Charité. Cinisello Balsamo : Silvana Editoriale, 2007.

17. - HUEBER, Régis. «Auguste Bartholdi. Le projet de phare pour Suez et la genèse de la statue de la Liberté ». Dans La Liberté éclairant le monde. Inventaire des collections historiques (1869-1904) du musée Bartholdi à Colmar. Vol. I, texte introductif (en préparation).

18. - Lettres et journal du second voyage en Égypte (1869) d'Auguste Bartholdi adressés à sa mère, 13 avril 1869, Colmar, musée Bartholdi, cote VI 3 E.

19. - « Travaux du canal maritime de Suez - Vue du seuil d'El-Guisr ». L'Illustration, 14 juin 1862, p. 384 ; « Une tranchée dans le canal de Suez ». Dessin de Dominique Grenet tiré de l'album inédit de Narcisse Berchère et publié dans MERRUAU, Paul. «Une excursion au canal de Suez ». Le Tour $d u$ Monde. Nouveau journal des voyages, 1862, p. 9 ; « Aspect du chantier n ${ }^{\circ} 5$. Milieu du seuil d'ElGuisr » d'après les croquis envoyés par M. Roussin. Le Monde illustré, 15 novembre 1862 ; VIATOR. «Isthme de Suez ». L'Illustration, 14 février 1863 ; « The Suez Canal - View from the plateau of ElGuisr ». Harper's Weekly Magazine, 26 octobre 1867.

20. - TROGOFF-KERBIGOËT [Trogoff de Kerbiguet], Louis de. À travers mon époque, Satires, Poésies diverses, La question musicale. Paris: Challamel Aîné, 1874, p. 163-174. Voir le site: https:// gallica.bnf.fr/ark:/12148/bpt6k5719108d.r= [consulté le 01/02/2019].

21. - «Essai à la mine des carrières de l'Attaka »; "Percement de l'isthme de Suez - Le premier coup de pioche à Port-Saïd ». L'Illustration, 15 mai 1859.

22. - Voir « Drague à long couloir » et « Élévateur ». L'Exposition universelle de 1867 illustrée, 16 mai 1867, p. 117 ; «Le canal maritime à Kantara; vue prise pendant le travail des dragues et des élévateurs, d'après une photographie de M. Désiré ». L'Illustration, 11 septembre 1869, p. 169 ; «Les grandes dragues et les appareils élévateurs du canal de Suez, Prix de mécanique à l'Académie des Sciences. Dessin de M. Mallert ». L'Univers illustré, 1869, p. 409.

23. - LA BÉDOLLIÈRE, Émile de. De Paris à Suez. Souvenirs d'un voyage en Égypte. Paris: G. Barba, 1870, p. 25.

24. - ABOUT, Edmond. Le Fellah. Souvenirs d'Égypte. Paris : L. Hachette, 1869, p. 402. Voir le site : https://gallica.bnf.fr/ark:/12148/bpt6k62077112 [consulté le 01/02/2019].

25. - AUDOUARD, Olympe. Le Canal de Suez. Chapitre détaché d'un livre sur l'Égypte, qui paraîtra prochainement. Paris: E. Dentu, 1864, p. 6. Consultable sur Gallica: https://gallica.bnf.fr/ ark:/12148/bpt6k58053683 [consulté le 01/02/2019].

26. - DOMERGUE, Adrien. Simples notes de voyages. Gabon, Madagascar, Guyane. Paris : Impr. Paul Dupont, 1893, p.109-110. Voir le site: https:/gallica.bnf.fr/ark:/12148/bpt6k1058815/ f108.image [consulté le 01/02/2019].

27. - ALLEAUME, Ghislaine. « De la marche à la frontière. Aménagement du territoire et gestion des populations dans le Delta oriental de 1820 à 1880 ». Dans PIATON, Claudine. L'Isthme et l'Égypte au temps de la Compagnie universelle du canal maritime de Suez (1858-1956). Le Caire: Ifao, 2016, p. 269-270.

28. - CROSNIER LECONTE, Marie-Laure. «Monumental au fil du canal». Dans PIATON, Claudine. L'Isthme et l'Égypte au temps de la Compagnie universelle du canal maritime de Suez. Op. cit., p. 109-136.

29. - BOUDHAR, Yasmina. Mémoires partagées en Méditerranée autour de la patrimonialisation de la figure de Ferdinand de Lesseps. Mémoire de muséologie sous la direction de Michel Colardelle. Paris : École du Louvre, 2007.

30. - Voir VATIN, Jean-Claude. « Après l'orientalisme, l'Orient des Orientaux ? ». Dans POUILLON, François et VATIN, Jean-Claude (dir.). Après l'Orientalisme, l'Orient créé par l'Orient. Paris : Institut d'études de l'Islam et des sociétés du monde musulman/Karthala, 2011, p. 7.

31. - Rencontre des membres de l'association du Souvenir de Ferdinand de Lesseps et du canal de Suez avec le gouverneur de Port-Saïd le 16 novembre 2014 et présentation des planches illustrées du projet de statuaire réalisé par un artiste alexandrin. 
32. - CHRISTO. 500000 barrels structure: The Wall in Suez Canal, project for temporary wall between Israel and Egypt. 1967, crayon, peinture émail et craie, collection particulière.

33. - Voir SINOZICH, Gina. We are Sailing the Suez canal on Our Way to Australia. 2003, Australian National Maritime Museum Collection; HONG, Soun. Suez. Jan 27. 2011. Huile sur toile, Korean Museum Association.

\section{RÉSUMÉS}

Les dix années de chantier du canal de Suez (1859-1869) constituent un chapitre incontournable de l'histoire des représentations du canal de Suez et donnent lieu à une abondante production d'images et de textes, bien avant l'ouverture de l'immense tranchée. Toute une littérature viatique se développe, avec ses propres codes, pour restituer l'effervescence suscitée par les travaux. Elle témoigne des incroyables bouleversements géographiques qui s'opèrent et est largement imprégnée d'une dimension propagandiste visant à convaincre l'opinion de la réussite de l'œuvre pharaonesque. Elle participe de l'invention d'un nouvel espace oriental dont on tend à légitimer l'existence pour le bien de la nation égyptienne. Le chantier de creusement, qui transforme en profondeur une région désertique aux confins de l'Égypte, représente une nouvelle frontière à apprivoiser et incarne plus concrètement encore la limite d'un monde et le début d'un autre, plus lointain. Dès l'annonce du percement de l'isthme, toute une symbolique propre aux espaces de limite et de fin géographiques se met en place et promet la réunion des peuples. Il finit surtout par incarner une entreprise de conquête où la victoire scientifique et technique de l'Occident sera célébrée en grande pompe au terme des dix années de travaux de creusement. Il demeure par contre un territoire habité par une population cosmopolite qui a produit un patrimoine métissé et une mémoire partagée.

The ten years it took to dig the Suez canal, from 1859 to 1869 , contribute to a founding chapter in the history of representations of the canal, leaving rich collections of pictures and texts, well before the canal's inauguration. A literary style emerged with its own specific codes, accompanying the work in progress on the site and depicting its effervescence. This literature bears witness to the profound geographical upheavals the canal would bring about and is usually inspired by propaganda considerations, aiming to convince the public of the imminent success of the work, undertaken on a truly pharaonic scale. It also contributes to the invention of the notion of a new oriental space, given legitimate existence for the sake of the Egyptian nation. The excavation work on the canal brought radical changes to a desert region at the edge of this nation, a new frontier to be tamed. It was the incarnation of the end of one world and the beginning of another one, beyond. As soon as the project for cutting through the isthmus was announced, symbolic representations developed to speak of limits and geographical ends, promising the coming together of peoples. Finally, the canal also came to embody an enterprise of conquest, a scientific and technical victory for the Western world, celebrated with much pomp and circumstance after the ten years' work. But the region remained a territory inhabited by a cosmopolitan population which has left a mixed, cross-cultural heritage and a divided memory. 
INDEX

Keywords : Suez canal, symbolism, orientalism, representations, discourse, ideology, art, travellers' tales, memory, heritage, work site, isthmus, geographical revolution, divided territory, frontier, limit

Mots-clés : canal de Suez, symbolique, orientalisme, représentations, discours, idéologie, art, récits de voyage, mémoire, patrimoine, chantier, isthme, révolution géographique, territoire partagé, frontière, limite

\section{AUTEUR}

\section{HÉLÈNE BRAEUNER}

Docteur en histoire de l'art, membre de l'EA 3400-ARCHE, Université de Strasbourg helene.braeuner@orange.fr 